\title{
Influence of Inhalation Injury on Incidence, Clinical Profile and Recovery Pattern of Dysphagia Following Burn Injury
}

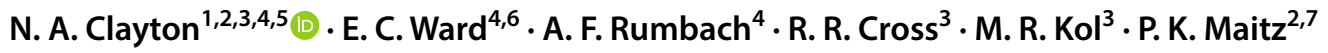

Received: 29 October 2019 / Accepted: 17 February 2020 / Published online: 27 February 2020

(c) Crown 2020

\begin{abstract}
Inhalation injury is predictive of dysphagia post burns; however, the nature of dysphagia associated with inhalation burns is not well understood. This study describes the clinical profile and recovery pattern of swallowing following inhalation burn injury. All patients admitted 2008-2017 with confirmed inhalation burns on laryngoscopy and managed by speech-language pathology (SLP) were included. Initial dysphagia presentation and dysphagia recovery pattern were documented using the FOIS. Co-presence of dysphonia was determined clinically and rated present/absent. Persistent laryngeal/pharyngeal injury at 6 months was documented using laryngoscopy. Data were compared to published data from a large adult burn cohort. All patients with confirmed inhalation burns during the study period received SLP input, enabling review of 38 patients $(68 \%$ male; $m=40.8$ years). Percent Total Body Surface Area burn ranged $1-90 \%, 100 \%$ had head and neck burns, $97 \%$ required mechanical ventilation (mean 9.4 days), $18 \%$ required tracheostomy and $100 \%$ had dysphonia. Comparing to non-inhalation burn patients, the inhalation cohort had significantly $(p<0.01)$ higher dysphagia incidence $(89.47 \%$ vs $5.6 \%)$; more with severe dysphagia at presentation ( $78.9 \%$ vs $1.7 \%$ ); increased duration to initiate oral intake ( $m=24.69$ vs 0.089 days); longer duration of enteral feeding ( $m=45.03$ vs 1.96 days); and longer duration to resolution of dysphagia ( $m=29.79$ vs 1.67 days). Persistent laryngeal pathology was present in $47.37 \%$ at 6 months. This study shows dysphagia incidence in burn patients with inhalation injury is 16 times greater than for those without inhalation injury. Laryngeal pathology due to inhalation injury increases dysphagia severity and duration to dysphagia recovery.
\end{abstract}

Keywords Deglutition $\cdot$ Deglutition disorders $\cdot$ Inhalation burn $\cdot$ Incidence $\cdot$ Severity $\cdot$ Recovery

\section{Introduction}

N. A. Clayton

nicola.clayton@health.nsw.gov.au

1 Speech Pathology Department, Concord Repatriation General Hospital, Hospital Rd, Building 42, Concord, NSW 2139, Australia

2 Burns Unit, Concord Repatriation General Hospital, Concord, NSW, Australia

3 Intensive Care Unit, Concord Repatriation General Hospital, Concord, NSW, Australia

4 School of Health \& Rehabilitation Sciences, University of Queensland, St Lucia, QLD, Australia

5 Faculty of Health Sciences, University of Sydney, Camperdown, NSW, Australia

6 Centre for Functioning and Health Research, Queensland Health, Buranda, QLD, Australia

7 Faculty of Medicine, University of Sydney, Camperdown, NSW, Australia
Inhalational injury involves damage to the respiratory tract and lungs by heat, smoke and toxic inhalants [1] with incidence increasing with the size of the burn injury as well as the age of the patient $[2,3]$. Along with pulmonary complications, inhalation injury is responsible for up to $77 \%$ of all deaths following burn injury and is most often a consequence of carbon monoxide poisoning [4, 5]. Smoke inhalation is present in $2-30 \%$ of all flame burn injuries; however, mucosal injury at the level of the vocal folds as a result of inhaled heat is rare [6].

The management of the burn patient with concomitant inhalation injury is widely accepted to be complex. Inhalation injury is well known to be an independent risk factor for mortality and is also associated with significant morbidity, which may be observed as early or late sequelae [7, 8]. Immediately following inhalation injury, risk of respiratory distress and airway compromise frequently necessitate the 
need for intubation and mechanical ventilation. Patients with significant airway involvement of their burn often develop direct mucosal injury to the respiratory tract, mucosal sloughing, and inflammation (Fig. 1), potentially leading to airway obstruction and impaired gas exchange. These physiological changes in addition to higher fluid resuscitation requirements lead to an increased risk for pulmonary and airway oedema, acute respiratory distress syndrome (ARDS), ventilator associated pneumonia (VAP), multiple organ dysfunction syndrome (MODS) and therefore longer duration of mechanical ventilation and length of stay in the Intensive Care Unit (ICU) [9]. Potential late consequences of inhalation injury may also occur. These may evidence as scarring, contracture and stenosis or stricture of the mucosal tissue, with subsequent impacts on physiological function of those structures affected [10].

Due to the early and potentially long-term damage to the laryngeal and pharyngeal structures, dysphagia and dysphonia are not uncommon in this population. It is already known that dysphagia is a common consequence of burn injury ( \pm inhalation injury), with the prevalence rate of dysphagia following thermal burn injury in the adult population documented to be $11.18 \%$ in the general adult population [11] and up to $46.97 \%$ in the aged ( $\geq 75$ years) burn population [12]. A number of predictive factors for dysphagia development post burns have also been identified [11] and subsequently validated [13]. These factors, with consideration to advancing age, include the presence of head and neck burns, total burn surface area (TBSA) $\geq 18 \%$, need for ICU admission and mechanical ventilation, and presence of tracheostomyand inhalation injury. However, whilst inhalation injury has been identified as a predictor for dysphagia and its complications, the incidence and severity of dysphagia, and the pattern of recovery specifically in the subset of patients with burn injury and associated inhalation injury are not well understood.

Dysphagia following burn injury, not specifically with an inhalation component, has been characterised as presenting with deficits in both the motor as well as sensory aspects of swallowing function with onset evident early in the acute admission. In many cases, complete recovery can be achieved prior to hospital discharge [14, 15], although a small proportion of patients will experience more severe swallowing impairment with a lengthy and at times incomplete recovery $[14,16]$. Initiation of oral intake following burn injury has been documented to take longer in patients identified with dysphagia than in those without [14-23] with lengthy periods of enteral nutrition often necessary. Resolution of dysphagia after burn injury also follows a variable, and sometimes protracted period of recovery. Burn patients, identified with dysphagia, have been reported to take between 1 and 222 days to achieve their premorbid swallowing function as defined by reaching premorbid oral diet and fluids and no longer requiring the aid of supplementary nutrition, therapeutic or compensatory manoeuvres [14-16, 18, 20].

Examination of dysphagia severity in a large cohort ( $n=438$ ) of patients following thermal burn injury, of which $6.85 \%$ were identified as having concomitant inhalation injury, has revealed that $41 \%$ presented with severe dysphagia at the point of initial swallowing assessment [14]. However, no specific sub-analysis of those patients with or without inhalation injury was conducted to determine any differential patterns of dysphagia severity. Considering that increased dysphagia severity and duration to recovery has also been connected to other factors necessary to managing inhalation injury, including endotracheal tube size and duration of intubation and tracheostomy [11, 13, 24-27], it is possible that dysphagia severity in this cohort will be even higher than in the head and neck burn population. The application of enteral feeding following burn injury is also necessary, not only as an adjunct to managing nutritional sequelae associated with dysphagia alone but also to ensure adequate nourishment to facilitate wound healing, physical and psychological rehabilitation [28]. Duration of enteral feeding in the general thermal burns population has been
Fig. 1 Inhalation injury at the level of the larynx (a) and main carina (b)
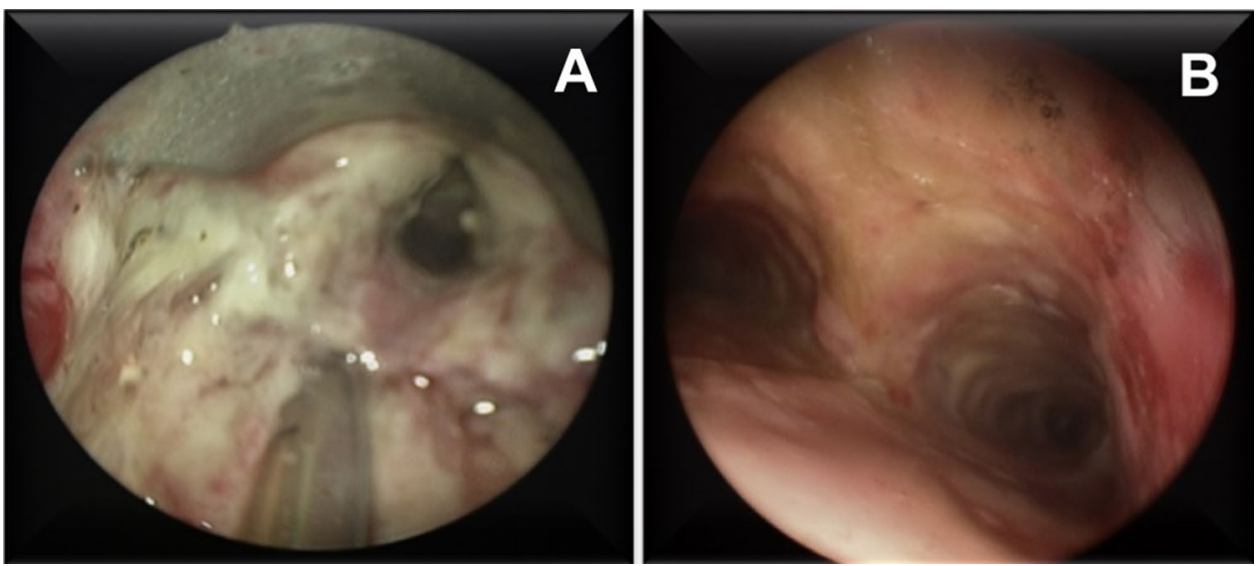
shown to be lengthy and often extends beyond the time that dysphagia has resolved [14-16, 23]. Again, there is high probability this also will be protracted in those with concomitant inhalation injury.

The presence of dysphagia post thermal burn injury has been reported to be connected with increased hospital length of stay, morbidity and mortality as well as requiring increased periods of Speech-Language Pathology (SLP) intervention $[12,14]$. Consequently, the literature to date clearly describes that dysphagia is a considerable issue with substantial contribution to the burden of treatment for a patient with thermal burn injury. Although data detailing the presentation and clinical progression of dysphagia for the general burn population are developing, and predictive factors including inhalation injury have been identified, clinical details regarding the incidence and clinical progression in the subset of patients who also present with inhalation injury are lacking. The aim of this study was therefore, to determine the incidence of dysphagia and describe the clinical profile of swallowing function for those admitted with inhalation burn injury, and contrast this with known patterns of recovery for those without inhalation injury post burn.

\section{Methods}

\section{Participants}

The model of care for severe burn injury is centralised to key specialist services within each state/territory in Australia. The NSW Statewide Burn Injury Service (SBIS) at Concord Repatriation General Hospital (CRGH) campus receives all patients in accordance with the Australian and New Zealand Burns Association (ANZBA) transfer guidelines, which includes those patients with cutaneous burn injury and concomitant inhalation burn injury. Within the SBIS at CRGH, SLP services are an integrated part of the multidisciplinary care team and a blanket referral system is in place to see all patients fulfilling a set of criteria inclusive of head and neck burns, suspected inhalation injury, ICU admission, mechanical ventilation, tracheostomy, concomitant neurological injury and age greater than 75 years. For the current study, all patients admitted to the SBIS at CRGH campus over a ten-year period (January 2008 to December 2017) for treatment of an acute thermal burn injury with the intention to treat being survival were identified through the SBIS database, then cross referenced with CRGH medical and SLP records. For inclusion in the study, patients must have had confirmed inhalation injury on laryngoscopy, had been assessed by the SLP service in accordance with the pre-existing blanket referral system, and have no prior medical record history of pre-existing burn injury, dysphagia, dysphonia, neurological or head and neck pathology. The study was conducted with full ethical clearance (CRGH Human Research and Ethics Committee: AU/1/7A68315).

\section{Procedure}

Information was obtained through a retrospective medical and SLP record review. For all eligible participants, information was collected from the time of initial injury and throughout the complete duration of inpatient treatment as well as outpatient care, until the time of dysphagia and dysphonia resolution or until swallowing and voice functioning had plateaued such that no further gains from treatment were anticipated. From the medical charts, key demographic and medical information was collected for all cases including age, gender, \% TBSA burn, mechanism of burn, anatomical location of burn, hospital length of stay (LOS), ICU LOS, days of mechanical ventilation and presence of tracheostomy.

Specific information pertaining to dysphagia, dysphonia and its recovery were then compiled from medical and SLP records. As the SLP service is an integrated part of the SBIS at CRGH in both the inpatient and outpatient setting, patients are reviewed on a daily basis initially and subsequently as clinically indicated until premorbid level of swallow and voice functioning is achieved or plateaued such that further gains are not anticipated.

\section{Assessment of Swallow Function}

As per the routine clinical procedures followed within this service swallowing function was assessed by a Clinical Swallowing Examination (CSE) within $24 \mathrm{~h}$ of admission for non-intubated patients or within $24 \mathrm{~h}$ post extubation if the patient were mechanically ventilated at the point of hospital admission. Following this initial CSE, regular reassessment occurred during the course of management until the patient was determined to have regained premorbid swallowing function. When required, patients within this burns service will be referred for a FEES assessment. FEES is conducted in preference to a videofluoroscopic assessment to minimise the need for transporting the patient to the VFSS suite and risk of infection. Factors currently limiting routine use of FEES assessment for patients within the burns unit are operational, created by staffing and equipment resource limitations.

The CSE assessments were conducted by one of two SLPs working in the services with 19 and 8 years of experience in burn injury management, respectively. The CSE procedure involved review of medical status, visual inspection of the oro-musculature and assessment of cranial nerves, then trials of selected fluid and food textures as clinically appropriate. The hyolaryngeal complex was 
palpated during each swallow and where feasible (considering skin integrity, etc.) and concurrent cervical auscultation was used as supplemental information to help inform the final clinical decision regarding swallow integrity. Appropriate diet and fluid consistencies along with other compensatory and therapeutic manoeuvres were recommended with the aim to rehabilitate swallowing function, minimise aspiration risk as well as maximise nutritional input. Clinical outcomes of the assessment were reported using the Functional Oral Intake Scale (FOIS). The FOIS [29] is a 7-point scale used to define dysphagia presence and severity, based on the degree of food and fluid modification as well as the need for enteral nutrition $(1=$ nothing by mouth; $3=$ tube dependent with consistent intake of food and fluid; $7=$ total oral diet with no restriction).

Throughout the admission, ongoing SLP swallowing management was delivered in accordance with individual patient needs and consistent with the clinical guidelines of Speech Pathology Australia [30]. Clinical practice remained standard and did not alter for the duration of this study. Conclusion of dysphagia treatment was deemed final once the SLP determined the patient's swallowing function had either reached premorbid status, or had plateaued (i.e. minimal changes noted and further advancement unlikely).

Several endpoints were computed to examine the recovery of swallowing function consistent with prior studies [14-18, 22, 23]. Days to initiate oral feeding (DIOF) was defined as the number of days between the time of hospital admission and commencing first oral intake. Days to resolution of dysphagia (DROD) was calculated as the number of days between hospital admission, resolution of premorbid swallowing function and resumption of premorbid oral diet and fluids without the aid of compensatory and/or therapeutic manoeuvres. Enteral feeding is a necessity in most patients with large burn injury due to the extremely high metabolic demands that ensue to combat catabolism and facilitate wound healing, hence it is not a surrogate marker of dysphagia in this clinical population. Days of enteral feeding (DOEF) was expressed as the number of days that enteral feeding was in place regardless of swallowing diagnosis or management.

Clinical outcomes data from the initial and discharge CSE were used to create a severity rating for dysphagia as per Rumbach et al. [14] where Mild Dysphagia was defined as requiring one level of restriction in either the food or fluid category (e.g. thin fluids and a soft dental diet) with the patient able to safely consume the majority of the modified meal; Moderate Dysphagia was defined as requiring one or more levels of restriction to both food and fluid consistencies to minimise aspiration risk (e.g. Mildly thick fluids and pureed solids) and the patient able to safely consume at least half of modified texture meal and Severe Dysphagia was defined as a high aspiration risk for all food and fluid consistencies with the patient being placed Nil By Mouth, with or without small trials of modified food or fluids only.

\section{Assessment of Laryngeal Pathology and Vocal Function}

Laryngeal/pharyngeal injury was documented using laryngoscopy, conducted routinely at clinically appropriate time points as determined by the managing SLP and/or ENT Surgeon until at least 6 months post injury, unless resolution of laryngeal pathology occurred earlier. Reports from each laryngoscopy were reviewed for salient features with presence of anatomical and physiological pathology noted. From the regular SLP assessments, the presence of dysphonia was routinely examined using perceptual assessment by the managing SLP and reported as a dichotomous rating (present/absent) throughout the complete duration of inpatient admission as well as outpatient treatment until at least 6 months post injury. Flexible Endoscopic Evaluation of Swallowing (FEES) was not routinely implemented at the time of laryngoscopy. This was due to either the acuity of the patient condition at the time of the procedure (e.g. laryngoscopy conducted in the emergency department for the primary reason of assessing airway patency and indication for intubation) or timing of procedure not permitting presence of a SLP to conduct FEES.

\section{Statistical Analyses}

All data were entered into an Excel database, then descriptive and inferential statistics were conducted using the Statistical Package for the Social Sciences (SPSS) Version 24.0. Data analysis took part in two stages. Initial analysis involved descriptive analysis of the present study's inhalation cohort (Inhal_1, $n=38$ ). Then any differences in the data between those patients with and without dysphagia within the inhalation injury cohort were examined.

In the second stage analysis, data from the current study's cohort were directly compared to previously published outcomes data from Rumbach et al. [14]. Access to the original data set for their 438 participants was provided with institutional ethical approval and consent by the study authors. The original data set was subdivided into two population subgroups for the current research: (a) those with inhalation injury (Inhal_2, $n=30$ ) and (b) a non-inhalation injury subset (Non-Inhal, $n=408$ ). Comparisons (Chi-square and $t$ tests) were conducted between the current and published inhalation cohorts, and between the current inhalation cohort and the published cohort with no inhalation injury. Significance was set at $p<0.05$. 


\section{Results}

\section{Inhalation Cohort}

Over the study period, 144 patients were admitted post thermal burn injury with suspected inhalation component, to the New South Wales SBIS CRGH Campus. Inhalation injury was confirmed on laryngoscopy in 38 participants (26 male; 12 female; mean age 40.82 years, range $17-71$, SD 14.30) who formed the study cohort. The percent total body surface area (TBSA) ranged from $1-90 \%$ (mean 35.28 , SD 23.55 ) with $100 \%$ exhibiting head and neck burns. Mechanical ventilation was required in $97 \%$ with a mean duration of 9.62 days ventilation (range $0-24$ days, SD 6.36) and 18\% required a tracheostomy. Hospital LOS ranged considerably from two and up to 213 days, with a mean of 60.2 days. On initial laryngoscopy (within $24 \mathrm{~h}$ of hospital admission), all participants exhibited evidence thermal injury to the pharyngeal and/or laryngeal mucosa. Laryngoscopy within one week of extubation or insertion of tracheostomy revealed persistent sloughy mucosal tissue in $8 \%$, significant oedema and erythema in $37 \%$, granulation or ulceration in $45 \%$ and restricted laryngeal movement in $45 \%$. Six months post injury, persistent laryngeal pathology was apparent on laryngoscopy in $47.37 \%$ of participants. This pathology specifically involved the presence of scar tissue with resultant contracture in varying degrees at the level of the true vocal cords, false vocal cords, aryepiglottic folds, and anterior and posterior glottis which restricted laryngeal function. In two cases, airway patency was also compromised to the degree that tracheostomy and laser cordotomy were necessary. All participants (100\%) exhibited dysphonia on clinical examination (Table 1).

Incidence of dysphagia was $89.47 \%$ in the cohort with inhalation injury. Mean DIOF was 24.69 days (range 1-200 days, SD 42.87). DROD was an average of 29.79 days (range 1-222 days, SD 41.18), whilst mean DOEF was 45.03 days (range $0-200$ days, SD 52.80). Dysphagia severity at the point of initial swallow assessment was severe in $78.9 \%$ of participants, moderate in $7.9 \%$, and mild in $2.6 \%$ with $10.5 \%$ exhibiting no clinical dysphagia at all. At the point of discharge from the acute care facility, $97.4 \%$ had completely recovered their swallow function to premorbid ability, whilst $2.6 \%$ continued to exhibit severe dysphagia with complete dependence on enteral nutrition. Analysis of all cases included in this study revealed a low incidence of instrumental assessments being conducted, with only 7 (18.4\%) participants undergoing a FEES procedure at some point during their admission. However, unfortunately as the timing of when this assessment occurred for each patient, and due to the low numbers of studies conducted, these data were not analysed or included in the current paper as it was deemed insufficient to make any conclusions or patterns regarding dysphagia presentation or recovery.

Comparison of participants in the inhalation cohort who were identified either with $(n=34)$ and without $(n=4)$ dysphagia is also reported in Table 1. Due to small and uneven group numbers, statistical comparisons were not conducted. However, patterns reveal those with dysphagia took longer to commence oral intake (mean 27.0 vs 6.5 days), longer to achieve premorbid swallowing function (mean $47.2 \mathrm{vs}$ 10.3 days) and had a greater duration of enteral feeding (mean 49.6 vs 10 days), compared to their non-dysphagic counterparts. Further to this, patients with dysphagia had
Table 1 Description of the total inhalation cohort, including sub-analysis of those with/ without dysphagia

\begin{tabular}{|c|c|c|c|c|c|c|}
\hline & \multicolumn{2}{|c|}{$\begin{array}{l}\text { Total inhalation cohort } \\
(n=38)\end{array}$} & \multicolumn{2}{|c|}{$\begin{array}{l}\text { Inhalation with dyspha- } \\
\text { gia }(n=34)\end{array}$} & \multicolumn{2}{|c|}{$\begin{array}{l}\text { Inhalation without } \\
\text { dysphagia }(n=4)\end{array}$} \\
\hline & $n(\%)$ & Mean (range) & $n(\%)$ & Mean (range) & $n(\%)$ & Mean (range) \\
\hline Age & - & $40.8(17-71)$ & - & $40.2(17-71)$ & - & $46.5(37-61)$ \\
\hline$\%$ TBSA burn & - & $35.3(1-90)$ & - & $26.8(1-90)$ & - & $22.5(4-63)$ \\
\hline Length of stay & - & $60.2(2-213)$ & - & $34.5(6-213)$ & - & $23.8(2-57)$ \\
\hline Endotracheal Intubation & $37(97.8)$ & $9.6(0-24)$ & $34(100)$ & $10.0(2-24)$ & $3(75)$ & $6.3(0-13)$ \\
\hline Tracheostomy & $7(18.4)$ & - & 7 (20.6) & - & $0(0)$ & - \\
\hline H\&N burn & $38(100)$ & - & $34(100)$ & - & $4(100)$ & - \\
\hline Mechanism of injury (flame) & $38(100)$ & - & $34(100)$ & - & $4(100)$ & - \\
\hline Dysphagia present & $34(89.5)$ & - & $34(100)$ & - & $4(100)$ & - \\
\hline DIOF & $38(100)$ & $24.7(1-200)$ & $34(100)$ & $27.0(3-200)$ & $4(100)$ & $6.5(1-13)$ \\
\hline DROD & $37(97.4)$ & $42.9(1-222)$ & $33(97.1)$ & $47.2(4-222)$ & $4(100)$ & $10.3(1-28)$ \\
\hline DOEF & $38(100)$ & $45.0(0-200)$ & 34 (100) & $49.6(3-200)$ & $4(100)$ & $10.0(0-28)$ \\
\hline Dysphonia present & $38(100)$ & - & $34(100)$ & - & $4(100)$ & - \\
\hline
\end{tabular}

TBSA total body surface area, DIOF days to initiate oral feeding, $D O E F$ days of enteral feeding, $D R O D$ days to resolution of dysphagia, ETT endotracheal tube, ICU intensive care unit, $L O S$ length of stay 
Table 2 Swallowing data across cohorts

\begin{tabular}{|c|c|c|c|c|c|}
\hline \multirow[t]{2}{*}{ Population Variable } & \multirow{2}{*}{$\begin{array}{l}\text { Inhal_1 } \\
\text { Mean (SD) }\end{array}$} & \multirow{2}{*}{$\begin{array}{l}\text { Inhal_2 } \\
\text { Mean (SD) }\end{array}$} & \multirow{2}{*}{$\begin{array}{l}\text { Non-Inhal } \\
\text { Mean (SD) }\end{array}$} & \multicolumn{2}{|c|}{ Comparisons } \\
\hline & & & & $\begin{array}{l}\text { Inhal_1 vs } \\
\text { Inhal_2 } \\
p \text { value }\end{array}$ & $\begin{array}{l}\text { Inhal_1 vs } \\
\text { Non-Inhal } \\
p \text { value }\end{array}$ \\
\hline Age & $40.82(14.30)$ & $40.40(18.58)$ & $38.17(17.32)$ & 0.917 & 0.085 \\
\hline TBSA & $35.28(23.55)$ & $30.70(19.49)$ & $8.97(9.43)$ & 0.394 & $<0.001$ \\
\hline ETT duration & $9.37(6.47)$ & $11.73(6.52)$ & $0.40(2.33)$ & 0.743 & $<0.001$ \\
\hline Hospital LOS & $60.21(51.37)$ & $54.40(42.54)$ & $14.47(15.33)$ & 0.620 & $<0.001$ \\
\hline ICU LOS & $16.08(21.05)$ & $16.77(11.14)$ & $0.51(3.162)$ & 0.872 & $<0.001$ \\
\hline DIOF & $24.69(42.87)$ & $19.67(22.59)$ & $0.089(5.48)$ & 0.084 & $<0.001$ \\
\hline DROD & $29.79(41.18)$ & $25.63(35.53)$ & $1.67(9.30)$ & 0.996 & $<0.001$ \\
\hline DOEF & $45.03(52.80)$ & $32.77(28.77)$ & $1.96(8.97)$ & 0.007 & $<0.001$ \\
\hline
\end{tabular}

Bold $=$ significant at $p<0.05$

$D I O F$ days to initiate oral feeding, $D O E F$ days of enteral feeding, $D R O D$ days to resolution of dysphagia, $E T T$ endotracheal tube, ICU intensive care unit, $L O S$ length of stay, TBSA total body surface area larger burn injuries (mean $36.8 \%$ vs $22.5 \%$ TBSA), a longer length of endotracheal intubation (mean 10.0 vs 6.3 days) and longer length of hospital stay (mean 34.5 vs 23.8 days) compared to those without dysphagia. Whilst it is well documented that intubation duration is associated with presence and severity of dysphagia, the results above suggest that there are additional factors beyond intubation duration that are associated with risk for dysphagia in the inhalation burn population. This will be further explored within the discussion section of the manuscript below.

\section{Comparison to Published Inhalation Cohort Data}

Comparing the current study's inhalation cohort (Inhal_1; $n=38$ ) to the 2012 published inhalation cohort (Inhal_2; $n=30$ ) of Rumbach and colleagues [14], all participants presented with burn injury to the head and neck region, with both cohorts comparable for age $(p=0.917)$ and size of burn injury $(p=0.394)$. Similarly, a high proportion of patients received mechanical ventilation $(97.8 \%$ vs $90 \%)$ with a comparable duration of intubation $(p=0.743)$. Further to this, the rate of tracheostomy insertion lacked significant variability at $18.4 \%$ and $20 \%$, respectively $(p=0.8686)$. Both hospital LOS as well as ICU LOS were again similar $(p=0.62$; $p=0.872$ ) across the two groups.

Importantly, the incidence of dysphagia in the current study (Inhal_1; 89.5\% dysphagic) was comparable $(p=0.7267)$ to the rate described by Rumbach et al. [14] (Inhal_2; 86.7\% dysphagic). Further to this, DIOF $(p=0.084)$ and DROD $(p=0.996)$ were also similar across the two cohorts. There was, however, a significant difference $(p=0.007)$ in DOEF between the two inhalation groups, with participants in the current study requiring a greater period of enteral feeding by approximately 13 days. These data are summarised in Table 2.

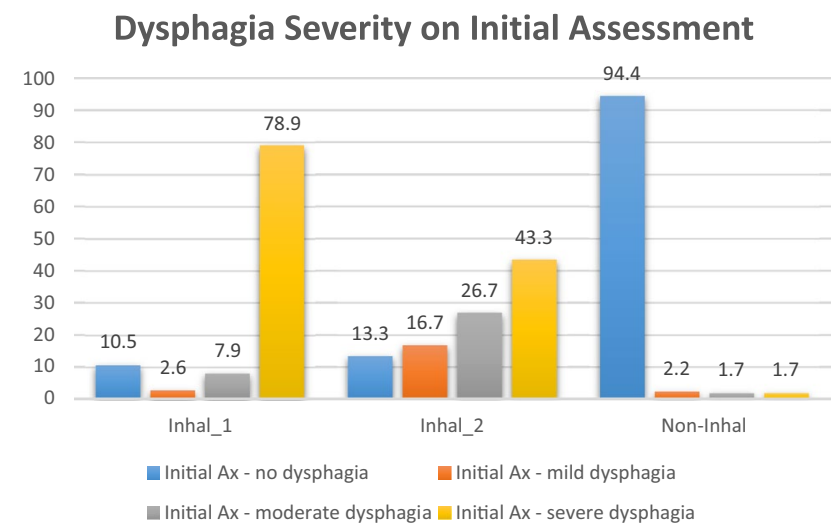

Fig. 2 Dysphagia severity on initial assessment across cohorts

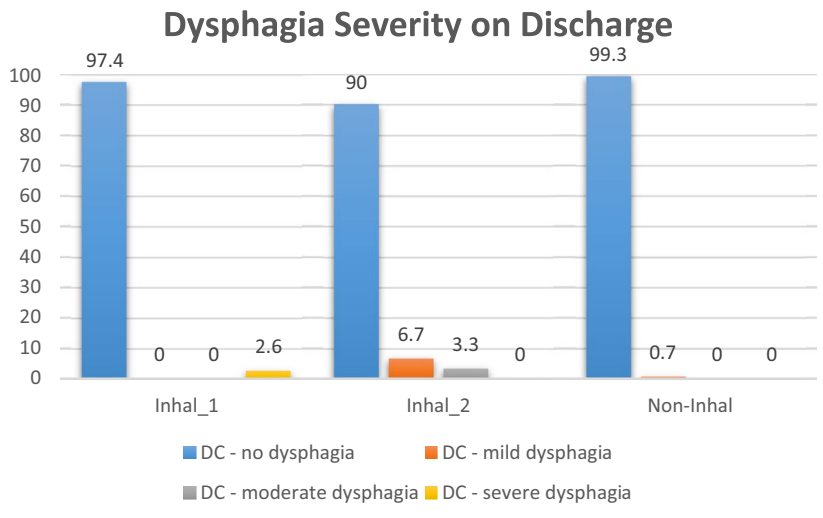

Fig. 3 Dysphagia severity on discharge across cohorts

Interestingly, dysphagia severity was significantly higher in the Inhal_1 cohort (78.9\% severe dysphagia) compared to the Inhal_2 cohort (43.3\% severe dysphagia) at the point of initial assessment $(p=0.0027)$; however, resolution was 
comparable at the point of discharge from the acute care facility ( $p=0.3772)$ (see Figs. 2 and 3 ).

\section{Comparison to Published Non-inhalation Cohort Data}

Comparisons between the inhalation cohort (Inhal_1; $n=38$ ) and the published non-inhalation cohort (NonInhal; $n=408$ ) of Rumbach et al. [14], revealed that age was comparable across the two groups. However, the proportion of head and neck burns (100\% Inhal_1 vs 26.2\% Non-Inhal; $p<0.0001)$ and rate of tracheostomy insertion (18.42\% Inhal_1 vs $0.49 \%$; Non-Inhal; $p<0.0001)$ were both significantly greater for those with inhalation injury. Furthermore, the inhalation cohort had a higher percentage TBSA burn $(p=0.000)$, longer duration of endotracheal intubation $(p=0.000)$, as well as greater hospital $(p=0.000)$ and ICU LOS $(p=0.000)$ than their non-inhalation injury counterparts.

Incidence of dysphagia was significantly greater $(p<0.0001)$ in participants with inhalation injury (Inhal_1; $89.47 \%$ dysphagic) compared to those without (Non-Inhal; $5.6 \%$ dysphagic). In addition, DIOF was significantly greater $(p<0.0001)$ in the current inhalation cohort (Inhal_1; mean DIOF $=24.69$ days) compared to those without inhalation injury (Non-Inhal; mean DIOF $=0.089$ days). Similarly, DROD was significantly elevated $(p<0.0001)$ in those with inhalation injury (Inhal_1; mean DROD $=29.79$ days) compared to those without (Non-Inhal; mean DROD $=1.67$ days). Lastly, DOEF was also significantly longer $(p<0.0001)$ in those with inhalation injury (Inhal_1; mean $\mathrm{DOEF}=45.03$ days) versus those without inhalation injury (Non-Inhal; mean DOEF $=1.96$ days).

Dysphagia severity was predictably greater $(p<0.0001)$ at initial assessment for the inhalation injury cohort in the present study (Inhal_1; 78.9\% severe dysphagia) compared to those without inhalation injury (Non-Inhal; $1.7 \%$ severe dysphagia) (Fig. 2); however, by the time of hospital discharge, the proportion of those with functional swallowing were analogous $(p=0.226)$ (Fig. 3).

\section{Discussion}

This study demonstrates that within our cohort of 38 participants, the presence of dysphagia is 16 times higher in patients with burn injury and a concomitant inhalation injury than those with no inhalation injury in the study of Rumbach et al. [14]. Further to this, laryngeal pathology due to inhalation injury is associated with an increase in dysphagia severity, with dysphagia recovery considerably protracted compared to their non-inhalation injury counterparts. The present data highlight that the SLP services need to be actively involved in the early and ongoing management of patients with inhalation injury in order to proactively support dysphagia risk and enhance swallow safety and recovery.

There are a number of potential precipitating factors behind the mechanism of dysphagia in this population. Firstly, a majority of patients (97.37\%) in the current study received intubation and mechanical ventilation with mean duration of 9.37 days, as part of their treatment. Rumbach and colleagues [13] have previously identified that the presence of ICU admission and mechanical ventilation hold positive predictive value at $56.82 \%$ and $59.92 \%$, respectively, for the development of dysphagia. Further to this, endotracheal intubation and mechanical ventilation is well recognised in the literature to be associated with dysphagia [31] with current incidence rates of dysphagia post extubation documented up to $62 \%$ [32]. Mechanisms for dysphagia in the intubated population have been proposed to be related to both the underlying medical diagnosis as well as the period of oropharyngeal muscle disuse and associated atrophy [33, 34]. Furthermore, the potential physiological and anatomical damage as a result of the endotracheal tube itself cannot be dismissed. The presence of muscle atrophy and localised endotracheal tube trauma is acknowledged to be probable in the present study population. However, given the rate of dysphagia following inhalation injury exceeds documented rates of dysphagia following endotracheal intubation, this suggests that there are additional processes that are impacting swallow integrity in this cohort.

A second causative factor for dysphagia in those patients with inhalation injury is the effect of the burn injury itself on oral, pharyngeal and laryngeal mucosa sensory and motor function. Histologically, whilst mucosa of the tongue blade, hard and soft palate appear to be resistant to the development of scar tissue and contractures following thermal inhalation or ingestion burn injury, the floor of mouth, pharyngeal and especially laryngeal mucosa are not [35]. As such, injury to these anatomical sites are associated with elevated risk for the development of scar tissue and contractures with subsequent impact upon motor and sensory function [36]. This may be postulated to represent as a reduction of airway protection during swallowing in addition to a reduction in airway responsiveness should an aspiration event occur. This is supported by the work of Clayton et al. [16, 24, 37] and Rumbach et al. [14, 18-20, 27] who have previously highlighted that oral, pharyngeal and laryngeal sensory deficits are common features of the burn injured patient with dysphagia.

Thirdly, the presence of acute deconditioning beyond the degree that is caused by presence of the endotracheal tube and associated oropharyngeal muscle disuse should not be underappreciated. Severe burn injury frequently induces a hypermetabolic state in burn sizes above 20 percent of the 
total body surface area, which can subsequently result in loss of muscle mass [28]. In the current study, the mean burn size of participants was $35.28 \%$, thus indicating that a substantial portion of participants in this cohort had a burn size that would result in catabolism, strongly inferring that they may be at risk for acute deconditioning including oropharyngeal muscle atrophy with its resultant effects on swallow physiology. This is also supported by previous work examining the profile of dysphagia following severe burn injury $[14,16-20$, 27], which documents that the primary characteristics of dysphagia following thermal burn injury include oral and pharyngeal phase deficits with the presence of reduced base of tongue retraction as well as impaired pharyngeal constriction that manifests as pharyngeal retention and elevated aspiration risk.

The extraordinarily high rate as well as greater severity of dysphagia in the present study highlights that early and rigorous dysphagia rehabilitation is paramount. Prevention and minimisation of the effects of deconditioning as well as contractures on swallow function is necessary to optimise functional swallowing outcomes. Evidence is emerging within the literature support this concept [16, 18-20]; however, further work is required to more clearly define optimal treatment methods to expedite safe transition to oral intake in this challenging population.

It is also important to consider that laryngeal pathology as a result of inhalation injury may not only adversely affect swallow function in the short term during the patient's immediate treatment post burn injury, but it may also compromise patient function later in life as a causative factor for the reduction of physiological reserve. Whilst the majority of patients who are cognitively and neurologically intact will learn to adapt to changes in laryngeal anatomy and physiology as it relates to swallowing, should an event occur to compromise that cognitive and neurological level of function, it is plausible to hypothesise that their ability to continue to adapt and compensate following an insult may be compromised.

One aspect that was not comparable between the two inhalation cohorts was the duration of enteral feeding. There are two possible explanations for this. Firstly, it is plausible that one cohort may have had issues with wound healing or increased nutritional needs for any one of a number of facets of burn rehabilitation, necessitating increased duration of supplementary nutrition. Secondly, given that these two cohorts were from two independent burn units, it is equally possible that habitual practices of each respective burn unit dietitian may vary slightly. As no specific nutritional parameters were recorded as a primary endpoint in this study, this aspect cannot be controlled for. The severity of dysphagia at the initial assessment of the 2 inhalation cohorts was also found to differ. As there was no significant difference in severity or injury presentation between the two cohorts, the reasons for this difference cannot be explained by the current data available.

\section{Limitations}

The current study is limited by the retrospective nature of the data collection, and as such, it is acknowledged that the data available was limited to those assessments conducted as part of routine clinical care. A prospective study of burn patients that includes set time points at which patients could complete a full battery of outcome measures pertaining to swallowing would be beneficial to fully appreciate the specifics regarding patterns of dysphagia recovery and presence of long-term laryngotracheal dysfunction. The small sample size is also a potential issue due to the known high level of heterogeneity in the clinical burn population. As this was a single Statewide service study, the need to involve multi-site data sets from other Statewide services to produce a more robust sample is recognised. However, given that characteristics of the current inhalation cohort did not differ significantly on most parameters from those with inhalation injury reported by Rumbach et al. [14], which was set in a separate Statewide service within the same country, the authors believe that patterns reported here are potentially true indications of the nature of recovery post inhalation burn injury.

There are also known issues with diagnosing patients with inhalation injury. The diagnosis itself of inhalational injury is often based on history and clinical suspicion [38]. In this study, however, a minimum of visual inspection via laryngoscopy was required to determine presence of inhalation injury. Despite this, it is accepted that this diagnostic process is subject to inter-observer variability and clinical experience. Various other modalities are also available to confirm the diagnosis, such as bronchoscopy, however, there are currently no agreed standard criteria for establishing the severity of this diagnosis.

Finally, there are multiple issues acknowledged regarding basing the diagnosis of dysphagia on a CSE alone. The authors fully acknowledge that a CSE does not provide the diagnostic information as available from instrumental assessments such as FEES or videofluoroscopy. Recognising the issues associated with a CSE, it is accepted that sub-clinical symptoms of dysphagia may have been missed, or severity of dysphagia not fully appreciated. As such the data presented within this study may be in fact an under-representation of the true incidence and severity of dysphagia. Future prospective studies incorporating regular instrumental assessment are needed to fully understand the influence of damage to the pharyngeal and laryngeal structures on swallow function. The authors also accept that there is debate regarding the added benefit of including cervical auscultation in the CSE and its value in informing 
on swallow integrity [39]. Auscultation was simply used in this study as an adjunct measure to add to the clinical information being collected on each patient and its limitations are acknowledged. The FOIS scale also has known limitations when applied in this population due to the long-term dependence of many patients on non-oral feeding for reasons other than dysphagia. However, the absence of population specific/sensitive tools limits choice of outcome measures at present.

\section{Conclusion}

The incidence of dysphagia in patients with inhalation injury is extremely high: 16 times greater than in those with burn injury but without an inhalation component in the present study. Laryngeal pathology due to inhalation injury increases not only dysphagia severity but also the duration to dysphagia recovery.

\section{Compliance with Ethical Standards}

Conflict of interest The authors declare that they have no conflict of interest.

\section{References}

1. Monteiro D, Silva I, Egipto P, Magalhães A, Filipe R, Silva A, Rodrigues A, Costa J. Inhalational injury in a burn unit: a retrospective review of prognostic factors. Ann Burns Fire Disasters. 2017;30(2):121-5.

2. Carr JA, Phillips BD, Bowling WM. The utility of bronchoscopy after inhalation injury complicated by pneumonia in burn patients: results from the National Burn Repository. J Burn Care Res. 2009;30:967.

3. Guo F, Chen XL, Wang YJ, Wang F, Chen XY, Sun YX. Management of burns of over $80 \%$ of total body surface area: a comparative study. Burns. 2009;35(2):210-4.

4. Ryan CM, Schoenfeld DA, Thorpe WP, Sheridan RL, Cassem EH, Tompkins RG. Objective estimates of the probability of death from burn injuries. N Engl J Med. 1998;338:362-6.

5. Darling GE, Keresteci MA, Ibañez D, Pugash RA, Peters WJ, Neligan PC. Pulmonary complications in inhalation injuries with associated cutaneous burn. J Trauma. 1996;40(1):83-9.

6. Gill P, Martin RV. Smoke inhalation injury. BJA Educ. 2015;15(3):143-8.

7. Colohan SM. Predicting prognosis in thermal burns with associated inhalational injury: a systematic review of prognostic factors in adult burn victims. J Burn Care Res. 2010;31(4):529-39.

8. Enkhbaatar P, Traber DL. Pathophysiology of acute lung injury in combined burn and smoke inhalation injury. Clin Sci. 2004;107(2):137-43.

9. Sutton T, Lenks I, Conrad P, Halrez M, Mosier M. Severity of inhalation injury is predictive of alterations in gas exchange and worsened clinical outcomes. J Burn Care Res. 2017;38(6):390-5.
10. Walker PF, Buehner MF, Wood LA, Boyer NL, Driscoll IR, Lundy JB, Cancio LC, Chung KK. Diagnoiss and management of inhalation injury: an updated review. Crit Care. 2015;19:351.

11. Rumbach AF, Ward EC, Cornwell PL, Bassett LV, Khan A, Muller MJ. Incidence and predictive factors for dysphagia after thermal burn injury: a prospective cohort study. J Burn Care Res. 2011;32(6):608-16.

12. Clayton NA, Nicholls CM, Blazquez K, Brownlow C, Maitz PK, Fisher OM, Issler-Fisher AC. Dysphagia in older persons following burn injury: burn location is irrelevant to risk of dysphagia and its complications in patients over 75 years. Burns. 2018;44:1997-2005.

13. Rumbach AF, Ward EC, Heaton S, Bassett LV, Webster A, Muller MJ. Validation of predictive factors of dysphagia risk following thermal burns: a prospective cohort study. Burns. 2014;40(4):744-50.

14. Rumbach AF, Ward EC, Cornwell PL, Bassett LV, Muller MJ. Clinical progression and outcome of dysphagia following thermal burn injury: a prospective cohort study. J Burn Care Res. 2012;33(3):336-46.

15. Ward EC, Uriarte M, Conroy A-L. Duration of dysphagic symptoms and swallowing outcomes after thermal burn injury. J Burn Care Rehabil. 2001;22(6):441-53.

16. Clayton NA, Ward EC, Maitz PKM. Intensive Swallowing and orofacial contracture rehabilitation after severe burn injury: a pilot study and literature review. Burns. 2017;43:e7-e17.

17. Clayton NA, Kennedy PJ, Maitz PKM. The Severe burns patient with tracheostomy: implications for management of dysphagia dysphonia and laryngotracheal pathology. Burns. 2010;36:850-5.

18. Rumbach AF, Ward EC, Zheng C, Cornwell P. Charting the recovery of dysphagia in two complex cases of post-thermal burn injury: physiological characteristics and functional outcomes. Speech Lang Hear. 2015;18(4):204-11.

19. Rumbach AF, Ward EC, Cornwell PL, Bassett LV, Muller MJ The challenges of dysphagia management and rehabilitation after extensive thermal burn injury: a complex case. J Burn Care Res. 2009;30(5):901-9055.

20. Rumbach AF, Ward EC, Cornwell PL, Bassett LV, Spermon ML, Plaza AL, et al. Dysphagia rehabilitation after severe burn injury: an interdisciplinary and multidisciplinary collaborative. J Med Speech-Lang Pathol. 2011;19(1):25-34.

21. Vo A, Kurmis R, Campbell J, Heath K, Greenwood J. Risk factors for and characteristics of dysphagia development in thermal burn injury and/or inhalation injury patients: a systematic review. Poster presentation at ANZBA ASM 2017.

22. Edelman DA, Sheehy-Deardorff DA, White MT. Bedside assessment of swallowing is predictive of an abnormal barium swallow examination. J Burn Care Res. 2008;29:89-96.

23. DuBose C, Groher MG, Carnaby-Mann GD, Mozingo DW. Pattern of dysphagia recovery after thermal burn injury. J Burn Care Rehabil. 2005;26(3):233-7.

24. Clayton NA, Ledgard J, Haertsch P, Kennedy PJ, Maitz PKM. Rehabilitation of speech and swallowing following post-burn reconstructive surgery to the lips and nose. J Burn Care Res. 2009;30:1039-45.

25. Smailes ST, Ives M, Richardson P, Martin RV, Dziewulski P. Percutaneous dilational and surgical tracheostomy in burn patients: incidence of complications and dysphagia. Burns. 2014;40(3):436-42.

26. Cheung W, Clayton NA, Milliss D, Li F, Tan J, Govindasamy K, Maitz P. The effect of endotracheal tube size on voice and swallowing outcomes in patients with thermal burn injury. Int J Speech Lang Pathol. 2013;15(2):216-20.

27. Rumbach AF, Ward EC, Cornwell PL, Bassett LV, Muller MJ. Physiological characteristics of dysphagia following thermal burn injury. Dysphagia. 2012;27(3):370-83. 
28. Rousseau AF, Losser MR, Ichai C, Beger MM. ESPEN endorsed recommendations: nutritional therapy in major burns. Clin Nutr. 2013;32(4):497-502.

29. Crary MA, Carnaby-Mann GD, Groher ME. Initial psychometric assessment of a Functional Oral Intake Scale (FOIS) for dysphagia in stroke patients. Arch Phys Med Rehabil. 2005;86:1516-9.

30. Speech Pathology Australia. Clinical Guideline: Dysphagia. Retrieved from https://speechpathologyaustralia.org.au/SPAweb/ Members/Clinical_Guidelines/SPAweb/Members/Clinical_Guide lines/Clinical_Guidelines.aspx?hkey=0fc81470-2d6c-4b17-90c0ced8b0ff2a5d. 2012

31. Brodsky MB, Gellar JE, Dinglas VD, Colantuoni E, MendezTellez PA, Shanholtz C, Palmer JB, Needham DM. Duration of endotracheal intubation is associated with dysphagia symptoms in acute ling injury patients. J Crit Care. 2014;29:574-9.

32. Skoretz SA, Flowers HL, Martino R. The incidence of dysphagia following endotracheal intubation: a systematic review. Chest. 2010;137:665-73.

33. Kress JP, Hall JB. ICU-acquired weakness and recovery from critical illness. New Engl J Med. 2014;370(17):1626-35.

34. Macht M, Wimbish T, Bodine C, Moss M. ICU-acquired swallowing disorders. Crit Care Med. 2013;41(10):2396-405.

35. Enoch S, Stephens P. Scarless healing: oral mucosa as a scientific model. Wounds UK. 2005;5(1):42-8.

36. Casper JK, Clark WR, Kelley RT, Colton RH. Laryngeal and phonatory status after burn/inhalation injury: a long term follow-up study. J Burn Care Rehabil. 2002;23(4):235-43.
37. Clayton NA, Kennedy PJ. Management of firecracker induced oropharyngeal burns. Adv Speech-Lang Pathol. 2007;9(3):265-70.

38. Jones SW, Williams FN, Cairns BA, Cartotto R. Inhalation injury: pathophysiology, diagnosis, and treatment. Clin Plast Surg. 2017;44(3):505-11.

39. Lagarde ML, Kamalski DM, van den Engel-Hoek L. The reliability and validity of cervical auscultation in the diagnosis of dysphagia: a systematic review. Clin Rehabil. 2016;30(2):199-207.

Publisher's Note Springer Nature remains neutral with regard to jurisdictional claims in published maps and institutional affiliations.

N. A. Clayton BAppSc Speech Pathology, MScMed, PhD

E. C. Ward BSpThy Hons, Grad Cert Ed, PhD

A. F. Rumbach BSc, MSpPathSt, GCHEd, PhD

R. R. Cross BSc Hons, MBBS, FCICM, Grad Dip Sim Ed

M. R. Kol BA, MBBS Hons1, FRACP, FCICM

P. K. Maitz MD, FRACS 